\title{
Towards High Performance CNT-Based Electrode Materials via Chemical Vapor Deposition Approach
}

\author{
Abdalghaffar M Osman', Ramadan A Geioushy ${ }^{2 *}$ and Osama A Fouad ${ }^{2}$ \\ ${ }^{1}$ Chemistry Department, King Fahd University of Petroleum and Minerals, Saudi Arabia \\ ${ }^{2}$ Nanomaterials and Nanotechnology Department, Advanced Materials Division, Central Metallurgical R \& D Institute (CMRDI), Egypt
}

*Corresponding author: Ramadan A Geioushy, Nanomaterials and Nanotechnology Department, Advanced Materials Division, Central Metallurgical R \& D Institute (CMRDI), PO Box, 87 Helwan, 11421 Cairo, Egypt.
Received Date: March 31, 2020

Published Date: April 06, 2020

\section{Opinion}

Recently, carbon nanotubes (CNTs) electrodes have increasingly attracted great attention in various applications such as energy storage, conductive adhesive; catalystsupports owing to their unique 1D tubular structure, high specific capacity, large surface area, and high electrical conductivity [1]. CNTs have high ability to mediate rapid electron transfer kinetics for a wide variety of electroactive species. Moreover, CNTs are easily modified and functionalized to suite different biological and environmental applications. However, the performance and the manufacture techniques of CNTs electrode have exposed to scientific researchers to be enhanced. CNTsbased electrodes could be fabricated via compressing and coating technologies. Unfortunately, these techniques have a negative impact on the adsorption performance of the electrode [2] as well as coating detachment after a short period [3]. At the present time, arc discharge, laser ablation, catalytic thermal decomposition and chemical vapor deposition (CVD) techniques have been used to synthesize CNTs $[4,5]$. Arc discharge and laser ablation are still not applicable in the industry due to the low mass production. Whereas, CVD performs a better production of large quantities of CNTs at a low cost, besides, the strong layer stuck over the substrate which in turn improves the performance of the electrode material and can be reused several times.

Most of the research in the field of electrochemistry that incorporate CNTs as electrode material focused on modifying a bare or a composite electrode by attaching CNTs through different methods. Among them, CNTs purchased from the market, dispersed with the aid of ultrasonication in acid or organic solvent for few minutes and the suspension is then deposited onto the surface of a bare or composite electrode and allowed to dry by evaporating the solvent at room temperature [6]. Electrodes prepared in this way are exposed to the detaching of CNTs from the surface. Another approach is to confine the CNTs on the surface of the bare or composite electrode by coating a mixture of CNTs and Nafion and allow the mixture to dry prior to use. A well-known method to immobilize CNTs on bare or composite electrodes is the electro polymerization of specific monomer from CNTs/monomer mixture. This method allowed fabricating different sensors and biosensors and finding a wide range of applications [7].

Vertically aligned CNTs arrays are considered among the best electrodes comprising CNTs. In addition to other advantages of CNTs, they provide an output current with many orders of magnitude larger than a single microelectrode. They have been widely employed in environmental and biological analyses. These types of electrodes are usually fabricated through the typical CVD or plasma-enhanced chemical vapor deposition (PECVD). For electrode fabrication, CNTs growth usually takes place on a metallic substrate like a thin layer of cobalt or Tantalum plate while different carbon sources can be used [8]. Very recently, CVD fabrication of CNTs/carbon cloth composite electrode has proved to enlarge the active surface area and decrease the electron transfer resistance [9].

We recently fabricated robust and long lasting CNTs based electrode via catalytic thermal decomposition of ethylene gas in a CVD reactor. The CNTs were grown on the surface of silver metal at $800{ }^{\circ} \mathrm{C}$. The prepared electrodes were used as indicator electrodes for differential electrolytic potentiometric determination of ascorbic acid in different complex matrices. These electrodes exhibited high efficiency in terms of reproducibility, sensitivity, and durability. The fabricated CNTs modified silver electrodes offered high surface area for the reaction and attained the equilibrium in very short times. 
They could able to determine ascorbic acid at concentrations less than $25 \mathrm{mM}$ [10]. The prepared electrode showed a stable response signal after using it many times for more than 10 weeks as shown in Figure 1. These promising results have encouraged us to fabricate CNTs modified electrodes through CVD for the sensing of different analyses and to employ them as detectors in flowing systems. The SEM image, Figure 2, of the electrode surface, exhibited the presence of bundles of aligned CNTs, which enhance the electrode performance as above mentioned for microelectrode arrays. CVD methods offer good opportunities to fabricate stable, good quality and long-lasting CNTs based electrodes with enhanced properties. Such electrodes can be used for daily routine analysis. CNTs can be synthesized on the surface of a suitable substrate in a temperature range of $600-850^{\circ} \mathrm{C}$ via CVD methods based on the catalyst and the used carbon source. The produced CNTs that are well adhesive to the substrate can be furthermore functionalized according to the application. Based on the recent literature and work done in our labs, we assume that CVD can play an important role to design electrodes modified with CNTs.

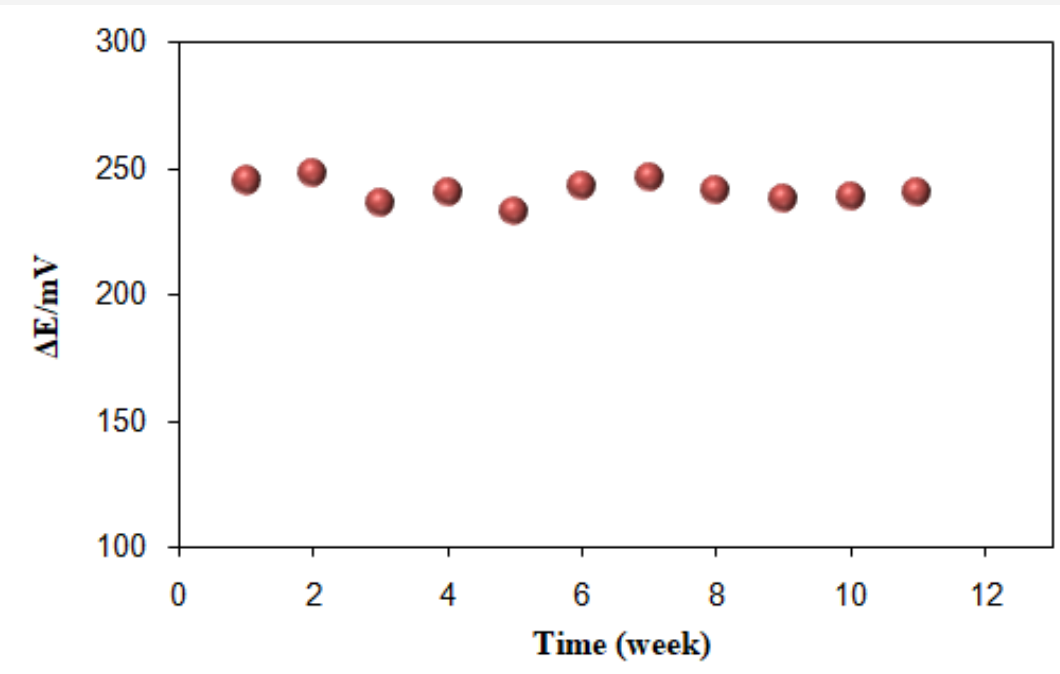

Figure 1: Response of the as-synthesized CNTs/Ag electrode for ten weeks.

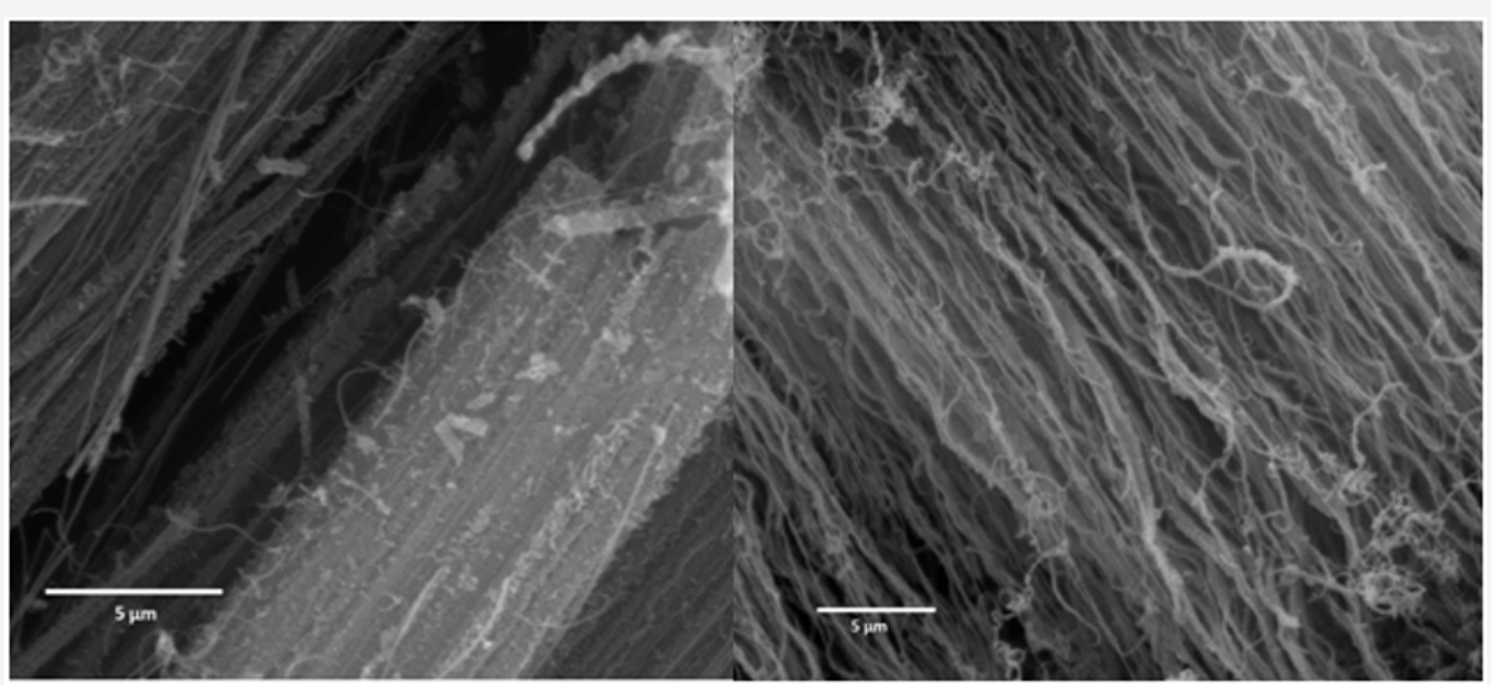

Figure 2: FE-SEM images of the as-synthesized CNTs via CVD technique.

\section{Acknowledgement}

We would like to thank King Fahd University of Petroleum and Minerals for availing scientific e-resources.

\section{Conflict of Interest}

No conflict of interest.

\section{References}

1. S Zhang, Y Wang, X Han, Y Cai, S Xu (2018) Optimizing the fabrication of carbon nanotube electrode for effective capacitive deionization via electrophoretic deposition strategy. Prog Nat Sci Mater Int 28(2): 251257.

2. BJ Kim, JS Park, YJ Hwang, JS Park (2016) Characteristics of copper meshes coated with carbon nanotubes via electrophoretic deposition. Appl Surf Sci 380: 2-7. 
3. Deng C, Jiang J, Liu F, Fang L, Wang J, et al. (2016) Influence of carbon nanotubes coatings onto carbon fiber by oxidative treatments combined with electrophoretic deposition on interfacial properties of carbon fiber composite. Appl Surf Sci 357 Part A: 1274-1280.

4. XM Liu, ZD Huang, SW Oh, B Zhang, PC Ma, et al. (2012) Carbon nanotube (CNT)-based composites as electrode material for rechargeable Li-ion batteries: A review. Compos Sci Technol 72(2): 121-144.

5. OA Fouad (2015) Promising method for production of carbon nanotubes from agricultural waste. EG26941.

6. H Gergeroglu, S Yildirim, MF Ebeoglugil (2020) Nano-carbons in biosensor applications: an overview of carbon nanotubes (CNTs) and fullerenes $\left(\mathrm{C}_{60}\right)$. SN Appl Sci 2: 603.

7. MM Barsan, ME Ghica, CMA Brett (2015) Electrochemical sensors and biosensors based on redox polymer/carbon nanotube modified electrodes: A review. Anal Chim Acta 881: 1-23.
8. R Chen, Y Li, K Huo, PK Chu (2013) Microelectrode arrays based on carbon nanomaterials: Emerging electrochemical sensors for biological and environmental applications. RSC Adv 41: 18698-18715.

9. Hu Z, Peng W, Tian W, Wnag F, Kang X, et al. (2019) A general strategy for in-situ fabrication of uniform carbon nanotubes on three-dimensional carbon architectures for electrochemical application. Appl Surf Sci 496: 143704 .

10. AM Osman, AM Abulkibash, MA Atieh (2020) Time-biased square wave differential electrolytic potentiometry for determination of ascorbic acid in a complex matrix at multi-walled carbon nanotubes modified silver electrodes. Arab J Chem 13(1): 2955-2963. 